\title{
Evaluation of Ellipsoid Zone Changes in Different Types of Diabetic Macular Edema After Intravitreal Bevacizumab Injection
}

Utku Limon ( $\square$ utku_limon@hotmail.com )

Umraniye EAH https://orcid.org/0000-0002-4998-1896

\section{Betul Ilkay Sezgin Akcay}

${ }^{1}$ University of Health Sciences Umraniye Training and Research Hospital

\section{Research Article}

Keywords: Ellipsoid zone changes, diffuse diabetic macular edema, cystoid diabetic macular edema, serous retinal detachment, intravitreal bevacizumab,

Posted Date: December 21st, 2021

DOI: https://doi.org/10.21203/rs.3.rs-1011438/v1

License: (c) (i) This work is licensed under a Creative Commons Attribution 4.0 International License.

Read Full License 


\section{Abstract}

Purpose: To evaluate the ellipsoid zone (EZ) changes according to the types of diabetic macular edema (DME) on optical coherence tomography (OCT) after intravitreal bevacizumab injection.

Methods: In this retrospective study, medical records of the patients who had treatment-naive DME and underwent intravitreal bevacizumab treatment between January 2015 and December 2021 were analyzed. Patients who meet the inclusion criteria and completed the follow-up period of 12 months were divided into 4 groups according to the types of diabetic macular edema on OCT (Diffuse DME [Group-1], cystoid DME [Group-2], diffuse DME with serous retinal detachment (SRD) [Group-3] and cystoid DME with SRD [Group-4]). Primary outcomes were EZ changes between the groups.

Results: 125 eyes of 96 patients who fulfilled the inclusion criteria and completed the follow-up period were included in the study. There were no statistically significant differences in EZ improvements between the groups at 4, 6 and 12 months after treatment $(p=0,594, p=0,836, p=0,486, p=0,748$ respectively). The mean logMAR BCVA and CMT of all eyes showed significant improvement at 4, 6 and 12 months. When the mean CMT was compared between each groups, there were significant differences between the Group- 1 and Group- 4 at 4, 6 and 12 months after treatment. $(p<0,05)$

Conclusion: The BCVA gains and improvement in the CMT were maintained in all four DME types during the first year of intravitreal bevacizumab therapy. The improvement in EZ disruption was not depent on the type of DME and improved similarly in all groups.

\section{Introduction}

Globally, diabetic retinopathy is the leading cause of blindness among working-aged adults [1]. Approximately $35 \%$ of patients with diabetic retinopathy develop diabetic macular edema (DME) [2]. It is estimated that there will be about 21 million diabetic macular edema patients by 2035. Increased vascular endothelial growth factor (VEGF) after hyperglycemia leads to increased vascular permeability and results macular edema [3].

The methods used in the treatment of DME are laser, corticosteroids (dexamethasone and fluonisolon), anti-VEGF agents (bevacizumab, ranibizumab and aflibercept) and pars plana vitrectomy $[4,5,6]$.

Systemic glucose, hypertension and hyperlipidemia regulation, diet and exercise must be added to these treatments. Anti-VEGF agents have become the mainstay of treatment of DME currently [7]. Bevacizumab is the most readily available anti-VEGF for most patients because of socioeconomic reasons.

For the first time in 1999, Otani et al. [8] classified DME types according to OCT patterns as sponge-like swelling, cystoid macular edema (CME) and serous retinal detachment (SRD). In the following years, new DME classifications based on OCT were made in parallel with the developments in OCT technologies [910]. 
Ellipsoid zone and ELM integrity and DRIL, which are used to determine the visual prognosis of patients with diabetic macular edema, have been studied frequently in recent years [11-12]. However, the status of these OCT biomarkers in different types of DME has not been adequately studied. In this study we evaluated the $E Z$ changes according to the types of diabetic macular edema after intravitreal bevacizumab injection on optical coherence tomography.

\section{Material And Methods}

The protocol of the present study conformed to the Declaration of Helsinki. The protocol and design of this study was approved by University of Health Sciences Umraniye Training and Research Hospital. IRB approval number was B.10.1.THK.4.34.H.GP.0.01/286.

In this retrospective study, medical records of the type-2 diabetic patients who had treatment-naive DME and underwent 3 loading doses of intravitreal bevacizumab injections then with Pro-re-Nata (PRN) treatment protocol with intravitreal bevacizumab between January 2015 and December 2021 were analyzed. Patients who meet the inclusion criteria and completed the follow-up period of 12 months were divided into 4 groups according to the types of DME on optical coherence tomography. Eyes with diffuse DME included in Group-1, eyes with cystoid DME included in Group-2, eyes with diffuse DME with serous retinal detachment (SRD) included in Group-3 and eyes with cystoid DME with SRD included in Group-4.

\section{Inclusion criteria}

1-Patients with treatment-naive DME with non-proliferative diabetic retinopathy,

2-Patients with central macular thickness $(\mathrm{CMT})>300 \mu \mathrm{m}$,

3-Patients with a best corrected visual acuity (BCVA) between 0.05 and 0.6 decimals,

4-At least 1 year of follow-up period,

5-Patients with $\mathrm{HbA} 1 \mathrm{C}$ less than \%10.

\section{Exclusion criteria}

1- Other retinal diseases (age-related macular degeneration, retinal vein occlusion, retinal detachment, macular hole, juxtafoveal telangiectasia...),

2-Diabetic macular edema with epiretinal membrane and vitreomacular traction,

3- Presence of other ocular disease such as uveitis, ocular trauma, optic nerve disease or glaucoma,

4- Foveal hard exudate, scar and ischemia (foveal avascular zone>600 $\mu \mathrm{m}$ ),

5- Patients with inadequate imaging or clinical data, 
6- Vitreous hemorrhage and severe cataract,

7- Patients who had received previous intravitreal injections, lasers or vitreoretinal surgery.

A detailed medical history, age, gender, documentation of medications, hypertension, $\mathrm{HbA1C}$ levels, hyperlipidemia, best corrected visual acuity (BCVA), central macular thickness (CMT), DME pattern and EZ on optical coherence tomography (OCT), and intraocular pressure (IOP) at baseline, and at months 4, 6 and 12 after intravitreal bevacizumab treatment were collected from patients charts.

\section{Examinations}

At all visits, BCVAs of all patients were measured with Snellen charts, anterior segment and dilated posterior segment examinations were performed with slit-lamp biomicroscopy, and intraocular pressure (IOP) was measured with Goldman applanation tonometry. Optical coherence tomography (Optovue, RTVue 100, CA, USA) measurements were calculated using device-generated software and repeated at all visits. The EZ was demarcated as the hyper-reflective band above the RPE. The EZ integrity was evaluated using the gray-scale cross-sectional images through the fovea in the central $1 \mathrm{~mm}$ area. The structural integrity of EZ was categorized as intact or disrupted. Fundus fluorescein angiography imaging was performed at baseline and was repeated according to the physician discretion.

\section{Injections}

Written informed consent was obtained from all of the patients. All injections were performed under sterile conditions in operating room with topical anesthesia. Initially, the patients received a loading dose of three consecutive monthly bevacizumab injections than continued with PRN protocol.

Pan-retinal photocoagulation (PRP) was applied to the patients who developed proliferative diabetic retinopathy during the follow-up. Focal laser therapy was applied to all patients with clinically significant macular edema at 6 months and later.

\section{Re-treatment criteria}

The presence of new or persistent DME, intraretinal or subretinal fluid on OCT and visual acuity loss of 1 or more lines at monthly visits.

\section{Outcome measurements}

Primary outcomes was EZ changes between the groups at months 4, 6 and 12 . Secondary outcomes were changes in BCVA, CMT and complications from baseline between the groups at months 4, 6 and 12 .

\section{Statistical methods}

Snellen BCVA values were converted to logMAR for statistical analysis. Statistical Package for the Social Sciences (SPSS) 24.0 program was used for statistical analysis. While evaluating the study data, in addition to descriptive statistical methods (Mean, Standard Deviation, Median, Frequency, Ratio, Minimum, Maximum), Friedman Halton test was used to compare the changes seen over time according 
to positions, and Wilcoxon Signed Rank test was used to determine the measurement differences between positions. Cochran's Q Test was used to compare the changes of the classified measures, and the McNemar Test was used to determine the differences of the classified repeated measures. Significance was evaluated at $p<0.05$ levels.

\section{Results}

The medical records of 328 DME patients were reviewed. 125 eyes of 96 patients who fulfilled the inclusion criteria and completed the follow-up period of 12 months were included in the study. Baseline and demographic characteristics of the patients were given in Table-1. There were no significant differences in any baseline characteristics among the four groups except CMT. According to the pairwise comparisons, it was found that CMT in Group 1 was significantly lower than CMT in Group $4(p=0.009)$.

\section{Ellipsoid zone changes}

Ellipsoid zone disruption was showed statistically significant improvement at months 4, 6 and 12 from baseline in all groups after intravitreal bevacizumab treatment $(\mathrm{p}<0.05)$. Improvement rates in the EZ at 12 months were $9 / 13$ eyes (69.2\%) in Group-1, 13/18 eyes (72.2\%) in Group-2, 11/14 eyes (78.5\%) in Group-3, and 12/18 eyes (66.6\%) in Group-4. Ellipsoid zone improvement was highest in Group-2. However, when the EZ improvement was compared between each groups, there were no statistically significant differences between the groups at 4, 6 and 12 months after treatment $(p=0,594, p=0,836$, $p=0,486, p=0,748$ respectively). Ellipsoid zone changes were given in Table- 2 and Figure- 1 . Figure- 2 shows a patients OCT at months 4, 6 and 12 with EZ disruption in Group-4.

\section{Visual Outcomes}

The mean logMAR BCVA of all eyes showed significant improvement from 0.78 at the baseline to 0.48 , 0.49 , and 0.48 at 4,6 , and 12 months after treatment, respectively $(p<0.001$, all).

The values for the diffuse DME eyes (Group-1) showed significant improvement from 0.69 at the baseline to $0.43,0.47$, and 0.46 at 4,6 , and 12 months after treatment, respectively $(p<0.001$, all).

The values for the cystoid DME eyes (Group-2) showed significant improvement from 0.80 at the baseline to $0.48,0.49$ and 0.48 at 4,6 , and 12 months after treatment, respectively $(p<0.001$, all).

The values for the diffuse DME and SRD eyes (Group-3) showed significant improvement from 0.79 at the baseline to $0.49,0.50$, and 0.49 at 4,6 , and 12 months after treatment, respectively $(p<0.001$, all).

The values for the cystoid DME and SRD eyes (Group-4) showed significant improvement from 0.82 at the baseline to $0.50,0.49$, and 0.48 at 4,6 , and 12 months after treatment, respectively $(p<0.001$, all).

When the mean BCVA was compared between each groups, there were no differences between the groups at 4,6 and 12 months after treatment $(p=0.509, p=0.533, p=0.951, p=0.896$ respectively $)$. The changes in 
BCVAs were shown in Figure-3.

\section{Anatomical Outcomes}

The mean CMT of all eyes showed significant improvement from $472.67 \mu \mathrm{m}$ at the baseline to 357.37 $\mu \mathrm{m}, 360.94 \mu \mathrm{m}$, and $349.74 \mu \mathrm{m}$ at 4,6 , and 12 months after treatment, respectively $(\mathrm{p}<0.001$, all).

The value for the diffuse DME eyes (Group-1) showed significant improvement from $433.83 \mu \mathrm{m}$ at the baseline to $317.1 \mu \mathrm{m}, 326.3 \mu \mathrm{m}$, and $320.7 \mu \mathrm{m}$ at 4,6 , and 12 months after treatment, respectively $(\mathrm{p}<0.001, \mathrm{all})$.

The value for the cystoid DME eyes (Group-2) showed significant improvement from $480.7 \mu \mathrm{m}$ at the baseline to $366.5 \mu \mathrm{m}, 369.0 \mu \mathrm{m}$, and $358.3 \mu \mathrm{m}$ at 4,6 , and 12 months after treatment, respectively $(p<0.001$, all).

The value for the diffuse DME and SRD eyes (Group-3) showed significant improvement from $470.6 \mu \mathrm{m}$ at the baseline to $352.2 \mu \mathrm{m}, 359.6 \mu \mathrm{m}$, and $344.2 \mu \mathrm{m}$ at 4,6 , and 12 months after treatment, respectively $(p<0.001$, all).

The value for the cystoid DME and SRD eyes (Group-4) showed significant improvement from $503.7 \mu \mathrm{m}$ at the baseline to $391.6 \mu \mathrm{m}, 387 \mu \mathrm{m}$, and $373.9 \mu \mathrm{m}$ at 4, 6, and 12 months after treatment, respectively $(p<0.001$, all).

The mean reductions in CMT at 12 months from baseline were $113.1 \mu \mathrm{m}, 122.4 \mu \mathrm{m}, 126.4 \mu \mathrm{m}$, and 129.8 $\mu \mathrm{m}$ in the 4 groups, respectively. When the mean CMT was compared between each groups, there were significant differences between the Group-1 and Group-4 at at 4, 6 and 12 months after treatment. $(p<0,05)$. The changes in CMTs were shown in Figure-4.

\section{Number of injections}

The mean number of injections over 12 months was 7.4 in Group-1, 8.0 in Group-2, 7.5 in Group-3, and 8.1 in Group-4. There was significant difference between the Group-1 and Group-4 in terms of the mean number of injections $(p=0.045)$.

\section{Ocular complications}

No ocular or systemic serious side effects occurred in any patient.

\section{Discussion}

Diabetic macular edema is the most important cause of moderate vision loss in patients with diabetic retinopathy [13]. With the development of spectral domain (SD) OCT in 2006, morphological changes in retinal layers and DME began to visualise detailed [14]. Imaging studies with SD-OCT have shown that the EZ integrity is highly correlated with BCVA [15-16]. Arf et al. [17] reported that SRD is not a type of DME in their DME classification with spectral domain OCT, but it should be evaluated as a morphological 
finding accompanying DME. Similarly, in this study, we evaluated SRD as a morphological finding accompanying diffuse DME or cystoid DME and then we divided DME types into 4 groups and examined the EZ changes after intravitreal bevacizumab treatment in these 4 groups during 1-year follow-up. Our favorable results indicate ellipsoid zone disruption significantly improved and there were no statistically significant differences between the DME groups after treatment.

Seo et al. [18] classified SRD as DME type in their study. They reported that EZ damage was higher in eyes with SRD, SRD improved with more injections than other DME types, and BCVA at month 12 was worse than other DME types. They also reported, the diffuse DME type maintained a good response to intravitreal ranibizumab treatment in a fewer number of injections. According to our study results, ellipsoid zone disruption values was not significantly different between the 4 types of DME at baseline and months at 4, 6 and 12 after intravitreal bevacizumab treatment. There was a significant difference between the mean number of injections in 1 year in between Group-1 (7.4, injection) and Group-4 (8.1, injection). However, EZ and BCVA improvement rates were statistically similar at moths 4, 6 and 12 in these two groups and other groups.

Koytak et al. [19] studied the effect of a single intravitreal injection of bevacizumab on different types of DME (SRD, diffuse DME and cystoid DME). They reported that BCVA was not significantly different between groups but cystoid DME and SRD subtypes are associated with a greater reduction in the CMT than the diffuse DME subtype. Similarly, our findings demonstrate that the greatest reduction in CMT was in Group-4 (Cystoid DME and SRD, $129.8 \mu \mathrm{m}$ ) and was statistically significant compared to the reduction in CMT in Group-1 (Diffuse DME, $113.1 \mu \mathrm{m}$ ).

Kim and et al. [20]. compared the effects of intravitreal bevacizumab on different types of DME. They also used Otani classification with OCT. They reported, at 12 months, changes in CMT and BCVA from baseline were not significantly different between groups. In our study, we confirmed that BCVA improved significantly and the retinal thickness decreased significantly with repeated intravitreal bevacizumab injection in all DME groups and there were no significantly different between groups.

Chatziralli et al. [21] divided DME into three groups as diffuse DME, cystoid DME, and cystoid DME with SRD in their study. They reported, eyes with cystoid DME presented worse EZ defect size than eyes with diffuse DME or cystoid DME with SRD. They also reported, after treatment with intravitreal ranibizumab, improvement in EZ defect size was more evident in eyes with cystoid DME than other types. They explained this as, the cysts in cystoid macular edema applied mechanical pressure to the photoreceptors, and EZ was improved when the cysts regressed with treatment. As in our study, SRDs can also accompany diffuse DME. Our findings demonstrate that, although EZ improvement in Group-2 (Cystoid DME) and Group-3 (Diffuse DME +SRD) was slightly higher than in the other groups, there was no statistically significant difference between all groups.

The most important advantage of our study is that SRD is classified as a subtype of diffuse and cystoid DME, unlike the Otani ${ }^{8}$ classification of DME types in 1999. However, our study has some limitations. 
First, we did not evaluate the disorganization of retinal inner layers (DRIL), interdigitation zone (IDZ) and external limiting membrane (ELM) which were important in the improvement in BCVA. We did not examine the EZ changes after other intravitreal anti-VEGF treatments and intravitreal steroids. Another important limitation are that it is a retrospective study and relatively have small number of patients in each type of DME.

In conclusion, BCVA gains and improvement in the CMT were maintained in all four DME types during the first year of intravitreal bevacizumab therapy. The improvement in EZ disruption was not depent on the type of DME and improved similarly in all groups after treatment with intravitreal bevacizumab injection.

\section{Declarations}

Acknowledgments: None

Conflict of interest: We declare no conflict of interest.

Funding sources: This study did not have a financial support.

Author contributions: U.L. conceptualized and conducted the study. The final version of the study was reviewed and confirmed by all the authors.

\section{References}

1. Sun Z, Yang D, Tang Z et al (2021) Optical coherence tomography angiography in diabetic retinopathy: an updated review. Eye (Lond).35(1):149-161. https://doi.org/ 10.1038/s41433-02001233-y

2. Zhang B, Chou Y, Zhao X et al (2021) Early Detection of Microvascular Impairments With Optical Coherence Tomography Angiography in Diabetic Patients Without Clinical Retinopathy: A Metaanalysis. Am J Ophthalmol. 222:226-237. https://doi.org/ 10.1016/j.ajo.2020.09.032

3. Mansour SE, Browning DJ, Wong K et al (2020) The Evolving Treatment of Diabetic Retinopathy. Clin Ophthalmol.14:653-678. https://doi.org/ 10.2147/OPTH.S236637

4. Yoshida S, Murakami T, Nozaki M et al (2021) Review of clinical studies and recommendation for a therapeutic flow chart for diabetic macular edema. Graefes Arch Clin Exp Ophthalmol. 259(4):815836. https://doi.org/ 10.1007/s00417-020-04936-w

5. Gurreri A, Pazzaglia A (2021) Diabetic Macular Edema: State of Art and Intraocular Pharmacological Approaches. Adv Exp Med Biol. 1307:375-389. https://doi.org/ 10.1007/5584_2020_535

6. Mirshahi R, Falavarjani KG, Molaei S et al (2021) Macular microvascular changes after intravitreal bevacizumab injection in diabetic macular edema. Can J Ophthalmol. 56(1):57-65. https://doi.org/ 10.1016/j.jcjo.2020.07.004

7. Mukai R, Matsumoto H, Akiyama H (2021) Surgical outcomes of vitrectomy for intractable diabetic macular edema. Graefes Arch Clin Exp Ophthalmol. 259(2):363-368. https://doi.org/ 
8. Otani T, Kishi S, Maruyama Y (1999) Patterns of diabetic macular edema with optical coherence tomography. Am J Ophthalmol.127: 688-693. https://doi.org/ 10.1016/s0002-9394(99)00033-1

9. Helmy YM, Atta HR (2013) Optical coherence tomography classification of diabetic cystoid macular edema. Clin Ophthalmol. 7: 1731-1737. https://doi.org/ 10.2147/OPTH.S47987

10. Panozzo G, Cicinelli M, Augustin A et al (2020) An optical coherence tomography-based grading of diabetic maculopathy proposed by an international expert panel: The European School for Advanced Studies in Ophthalmology classification. Eur J Ophthalmol. 30(1):8-18. https://doi.org/ $10.1177 / 1120672119880394$

11. De S, Saxena S, Kaur A et al (2021) Sequential restoration of external limiting membrane and ellipsoid zone after intravitreal anti-VEGF therapy in diabetic macular oedema. Eye (Lond). 35(5):1490-1495. https://doi.org/ 10.1038/s41433-020-1100-0

12. Achiron A, Kydyrbaeva A, Man V et al (2017) Photoreceptor integrity predicts response to anti-VEGF treatment. Ophthalmic Res. 57:37-41. https://doi.org/ 10.1159/000448522

13. Williamson TH (2021) Artificial intelligence in diabetic retinopathy. Eye (Lond). 35(2):684. https://doi.org/ 10.1038/s41433-020-0855-7

14. Kwan CC, Fawzi AA (2019) Imaging and Biomarkers in Diabetic Macular Edema and Diabetic Retinopathy. Curr Diab Rep. 19(10):95. https://doi.org/ 10.1007/s11892-019-1226-2

15. Eraslan S, Yıldırım Ö, Dursun Ö et al (2020) Relationship Between Final Visual Acuity and Optical Coherence Tomography Findings in Patients with Diabetic Macular Edema Undergoing Anti-VEGF Therapy. Turk J Ophthalmol (3):163-168. https://doi.org/ 10.4274/tjo.galenos.2019.91962

16. Gerendas BS, Prager S, Deak G et al (2018) Predictive imaging biomarkers relevant for functional and anatomical outcomes during ranibizumab therapy of diabetic macular oedema. $\mathrm{Br} \mathrm{J}$ Ophthalmol. 102: 195-203. https://doi.org/ 10.1136/bjophthalmol-2017-310483

17. Arf S, Sayman Muslubas I, Hocaoglu M et al (2020) Spectral domain optical coherence tomography classification of diabetic macular edema: a new proposal to clinical practice. Graefes Arch Clin Exp Ophthalmol. 258(6):1165-1172. https://doi.org/ 10.1007/s00417-020-04640-9

18. Seo KH, Yu SY, Kim M et al (2016) Visual and morphologic outcomes of intravitreal ranibizumab for diabetic macular edema based on optical coherence tomography patterns. Retina. 36:588-595. https://doi.org/ 10.1097/IAE.0000000000000770

19. Koytak A, Altinisik M, Sogutlu Sari E et al (2013) Effect of a single intravitreal bevacizumab injection on different optical coherence tomographic patterns of diabetic macular oedema. Eye. 27:716-721. https://doi.org/ 10.1038/eye.2013.17

20. Kim M, Lee P, Kim Y et al (2011) Effect of intravitreal bevacizumab based on optical coherence tomography patterns of diabetic macular edema. Ophthalmologica.226(3):138-44. https://doi.org/ $10.1159 / 000330045$

21. Chatziralli I, Theodossiadis G, Dimitriou E et al (2020) Association between the patterns of diabetic macular edema and photoreceptors' response after intravitreal ranibizumab treatment: a spectral- 
domain optical coherence tomography study. Int Ophthalmol. 40(10):2441-2448. https://doi.org/ $10.1007 /$ s10792-020-01423-3

\section{Tables}

Table-1: Baseline and demographic characteristics of the patients 


\begin{tabular}{|c|c|c|c|c|c|}
\hline & $\begin{array}{l}\text { Group- } \\
\text { (Diffuse DME) }\end{array}$ & $\begin{array}{l}\text { Group-2 } \\
\text { (Cystoid } \\
\text { DME) }\end{array}$ & $\begin{array}{l}\text { Group-3 } \\
\text { (Diffuse DME } \\
\text { and SRD) }\end{array}$ & $\begin{array}{l}\text { Group-4 } \\
\text { (Cystoid DME } \\
\text { and SRD) }\end{array}$ & $p$ value \\
\hline Female & $14(\% 46,7)$ & $15(\% 45,5)$ & $14(\% 45,2)$ & $16(\% 51,6)$ & \multirow[t]{2}{*}{${ }^{a} 0,953$} \\
\hline Male & $16(\% 53,3)$ & $18(\% 54,5)$ & $17(\% 54,8)$ & $15(\% 48,4)$ & \\
\hline $\begin{array}{l}\text { Age, year } \\
(\text { Mean } \pm S D)\end{array}$ & $54,23 \pm 6,66$ & $55,12 \pm 5,66$ & $54,42 \pm 6,79$ & $54,29 \pm 5,05$ & ${ }^{b} 0,931$ \\
\hline $\begin{array}{l}\text { DM duration, year } \\
\text { (Mean } \pm \text { SD) }\end{array}$ & $22,07 \pm 6,37$ & $21,42 \pm 4,65$ & $21,10 \pm 3,94$ & $21,06 \pm 4,07$ & ${ }^{b} 0,840$ \\
\hline \multicolumn{6}{|l|}{ DM treatment } \\
\hline$O A D, n(\%)$ & $4(\% 13,3)$ & $3(\% 9,1)$ & $3(\% 9,7)$ & $4(12,9)$ & \multirow[t]{2}{*}{${ }^{a} 0,930$} \\
\hline Insulin, n (\%) & $26(\% 86,7)$ & $30(\% 90,9)$ & $28(90,3)$ & $27(87,1)$ & \\
\hline $\begin{array}{l}\text { HbA1C (Mean } \pm \\
S D)\end{array}$ & $7,25 \pm 0,89$ & $7,15 \pm 0,87$ & $7,24 \pm 0,84$ & $7,01 \pm 0,61$ & ${ }^{b} 0,634$ \\
\hline $\begin{array}{l}\text { Hypertension, } n \\
\text { (\%) }\end{array}$ & $21(\% 70,0)$ & $25(\% 75,8)$ & $23(\% 74,2)$ & $24(\% 77,4)$ & ${ }^{a} 0,922$ \\
\hline $\begin{array}{l}\text { Hyperlipidemia, } n \\
\text { (\%) }\end{array}$ & $9(\% 30,0)$ & $9(\% 27,3)$ & $8(\% 25,8)$ & $7(\% 22,6)$ & ${ }^{a} 0,929$ \\
\hline $\begin{array}{l}\text { Hemodialysis, } n \\
\text { (\%) }\end{array}$ & $2(\% 6,7)$ & $2(\% 6,1)$ & $4(\% 12,9)$ & $4(\% 12,9)$ & ${ }^{a} 0,970$ \\
\hline \multicolumn{5}{|l|}{ Lens } & \multirow[t]{3}{*}{${ }^{a} 0,911$} \\
\hline Phakic, n (\%) & $25(\% 83,3)$ & $27(\% 81,8)$ & $24(\% 77,4)$ & $24(\% 77,4)$ & \\
\hline $\begin{array}{l}\text { Pseudophakic, n } \\
\text { (\%) }\end{array}$ & $5(\% 16,7)$ & $6(\% 18,2)$ & $7(\% 22,6)$ & $7(\% 22,6)$ & \\
\hline$B C V A$ Mean $\pm S D$ & $0,69 \pm 0,40$ & $0,80 \pm 0,36$ & $0,79 \pm 0,33$ & $0,82 \pm 0,35$ & ${ }^{b} 0,509$ \\
\hline $\begin{array}{l}\text { CMT Mean } \pm \\
S D\end{array}$ & $433,83 \pm 69,03$ & $480,70 \pm 89,32$ & $470,68 \pm 104,93$ & $503,71 \pm 66,05$ & ${ }^{b} 0,015^{*}$ \\
\hline \multicolumn{6}{|c|}{$\begin{array}{l}\text { DM: diabetes mellitus, OAD: oral anti-diabetics, BCVA: best corrected visual acuity, CMT: central } \\
\text { macular thickness, }\end{array}$} \\
\hline \multicolumn{6}{|c|}{$\begin{array}{l}\text { OCT: optical chorence tomography, ME: macular edema, SRD: serous retinal detachment, DME: } \\
\text { diabetic macular edema, }\end{array}$} \\
\hline \multicolumn{6}{|c|}{ apearson Chi-Square, bone-Way Anova, $p<0,05^{*}$} \\
\hline
\end{tabular}


Table-2: Ellipsoide zone changes

Page 12/15 


\begin{tabular}{|c|c|c|c|c|c|}
\hline & Baseline (EZ) & $\begin{array}{l}\text { Month } 4 \\
\text { (EZ) }\end{array}$ & $\begin{array}{l}\text { Month } 6 \\
\text { (EZ) }\end{array}$ & $\begin{array}{l}\text { Month } 12 \\
\text { (EZ) }\end{array}$ & Ip value \\
\hline \multicolumn{6}{|c|}{ Group-1 (Diffuse DME, $n=30$ ) } \\
\hline Intact, $n$ (\%) & $17(56,7)$ & $24(80)$ & $26(86,7)$ & $26(86,7)$ & $0,001^{\star \star}$ \\
\hline Disrupted, n (\%) & $13(43,3)$ & $6(20)$ & $4(13,3)$ & $4(13,3)$ & \\
\hline \multicolumn{6}{|c|}{ Group-2 (Cystoid DME, $n=33$ ) } \\
\hline & $15(45,5)$ & $24(72,8)$ & $25(75,8)$ & $28(84,9)$ & \\
\hline \multirow[t]{2}{*}{ Intact, $n(\%)$} & & & & & $0,001^{\star *}$ \\
\hline & $18(54,5)$ & $9(27,2)$ & $8(24,2)$ & $5(15,1)$ & \\
\hline \multicolumn{6}{|l|}{ Disrupted, $n(\%)$} \\
\hline \multicolumn{6}{|c|}{ Group-3 (Diffuse DME+SRD, $n=31$ ) } \\
\hline & $17(54,9)$ & $25(80,7)$ & $27(87,1)$ & $28(90,4)$ & \\
\hline \multirow[t]{2}{*}{ Intact, $n$ (\%) } & & & & & $0,001^{\star \star}$ \\
\hline & $14(45,1)$ & $6(19,3)$ & $4(12,9)$ & $3(9,6)$ & \\
\hline \multicolumn{6}{|l|}{ Disrupted, $n$ (\%) } \\
\hline \multicolumn{6}{|c|}{ Group-4 (Cystoid DME+SRD, n=31) } \\
\hline & $13(41,9)$ & $23(74,2)$ & $23(74,2)$ & $25(80,7)$ & \\
\hline \multirow[t]{2}{*}{ Intact, $n$ (\%) } & & & & & $0,001^{\star *}$ \\
\hline & $18(58,1)$ & $8(25,8)$ & $8(25,8)$ & $6(19,3)$ & \\
\hline Disrupted, $n$ (\%) & & & & & \\
\hline
\end{tabular}


[Cochran's Q Test, ${ }^{*} \mathrm{p}<0,01$

EZ: Ellipsoide zone, DME: Diabetic macular edema, SRD: serous retinal detachment

\section{Figures}

Figure-1:Ellipsoid zone disruption during 12 months

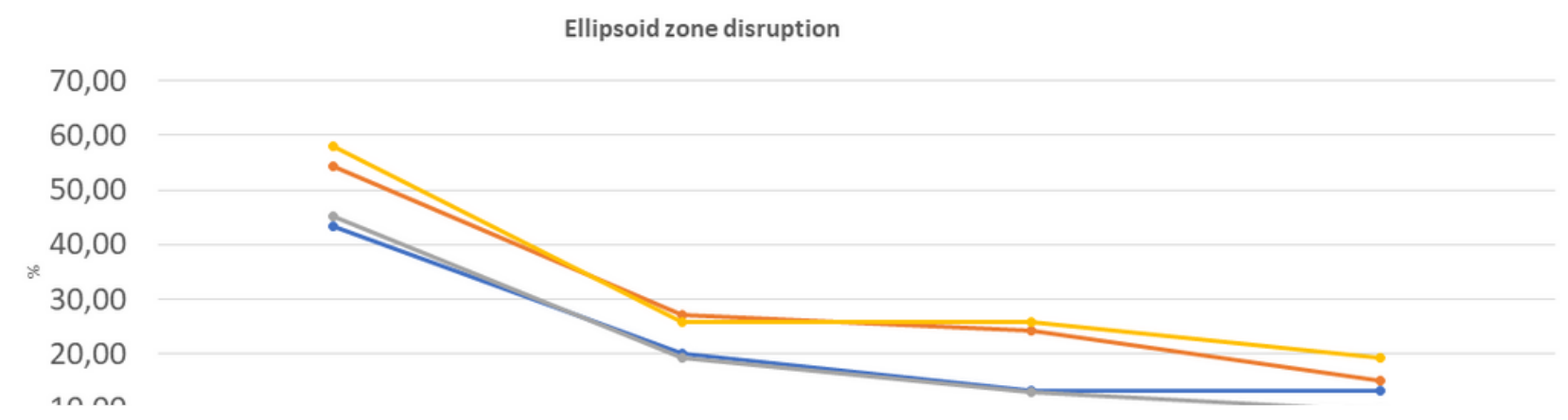

\section{Figure 1}

Ellipsoide zone distruption during 12 months.

\section{Figure 2}

Optical coherence tomography (OCT) imaging of a patient. (A) There is cystoid macular edema, serous retinal detachment, and ellipsoid zone disruption on baseline OCT. (B) At month 4, after 3 anti-VEGF injections, serous retinal detachment completely regressed, cystoid macular edema was significantly reduced, but ellipsoid zone disruption persists. (C) At month 6, serous retinal detachment and cystoid macular edema recurred. Ellipsoid zone disruption persists. (D) At month 12, after 7 intravitreal bevacizumab injections serous retinal detachment completely regressed, cystoid macular edema was significantly reduced, but ellipsoid zone disruption persists. 
Figure 3

Best corrected visual acuity changes.

\section{Figure 4}

Central macular thickness changes. 